\title{
Alendronate Can Improve Bone Alterations in Experimental Diabetes by Preventing Antiosteogenic, Antichondrogenic, and Proadipocytic Effects of AGEs on Bone Marrow Progenitor Cells
}

\author{
Sara Rocío Chuguransky, Ana María Cortizo, and Antonio Desmond McCarthy \\ Laboratorio de Investigaciones en Osteopatías y Metabolismo Mineral (LIOMM), Department of Biological Sciences, \\ School of Exact Sciences, National University of La Plata, Calle 47 y 115, 1900 La Plata, Argentina
}

Correspondence should be addressed to Antonio Desmond McCarthy; mccarthy@biol.unlp.edu.ar

Received 26 July 2016; Accepted 3 October 2016

Academic Editor: Pengjun Shi

Copyright (C) 2016 Sara Rocío Chuguransky et al. This is an open access article distributed under the Creative Commons Attribution License, which permits unrestricted use, distribution, and reproduction in any medium, provided the original work is properly cited.

\begin{abstract}
Bisphosphonates such as alendronate are antiosteoporotic drugs that inhibit the activity of bone-resorbing osteoclasts and secondarily promote osteoblastic function. Diabetes increases bone-matrix-associated advanced glycation end products (AGEs) that impair bone marrow progenitor cell (BMPC) osteogenic potential and decrease bone quality. Here we investigated the in vitro effect of alendronate and/or AGEs on the osteoblastogenic, adipogenic, and chondrogenic potential of BMPC isolated from nondiabetic untreated rats. We also evaluated the in vivo effect of alendronate (administered orally to rats with insulindeficient Diabetes) on long-bone microarchitecture and BMPC multilineage potential. In vitro, the osteogenesis (Runx2, alkaline phosphatase, type 1 collagen, and mineralization) and chondrogenesis (glycosaminoglycan production) of BMPC were both decreased by AGEs, while coincubation with alendronate prevented these effects. The adipogenesis of BMPC (PPAR $\gamma$, intracellular triglycerides, and lipase) was increased by AGEs, and this was prevented by coincubation with alendronate. In vivo, experimental Diabetes (a) decreased femoral trabecular bone area, osteocyte density, and osteoclastic TRAP activity; (b) increased bone marrow adiposity; and (c) deregulated BMPC phenotypic potential (increasing adipogenesis and decreasing osteogenesis and chondrogenesis). Orally administered alendronate prevented all these Diabetes-induced effects on bone. Thus, alendronate could improve bone alterations in diabetic rats by preventing the antiosteogenic, antichondrogenic, and proadipocytic effects of AGEs on BMPC.
\end{abstract}

\section{Introduction}

Bisphosphonates (BPs) have been extensively used to treat postmenopausal, age-related and steroid-induced osteoporosis, Paget's disease, and osteolysis-induced hypercalcemia associated with multiple myeloma and metastatic cancers [1]. The chemical structure of these drugs is similar to inorganic pyrophosphate: while the latter has a $\mathrm{P}-\mathrm{O}-\mathrm{P}$ bond linking both phosphate groups, in the former it is replaced by a $\mathrm{P}-\mathrm{C}-$ $\mathrm{P}$ structure. BPs possess a so-called "bone hook" consisting of both phosphonate groups, facilitating its binding to bone mineral [2]. Thus, BPs accumulate rapidly in bone tissue where they primarily inhibit the activity of bone-resorbing osteoclasts [1].

Nitrogen-containing bisphosphonates (N-BPs) such as alendronate are the most potent kind; they inhibit farnesyl pyrophosphate synthase (FPPS), a key regulatory enzyme in the mevalonate pathway, and thereby prevent prenylation of small GTPase signaling proteins. In bone, protein isoprenylation is essential for osteoclastogenesis and bone resorption [1, 3]. Thus, treatment with N-BPs is currently the most common treatment for postmenopausal osteoporosis [1, 3]. In vivo, treatment with BPs has an antifracture effect via inhibition of bone resorption and increase in bone mass [3]. 
Previous studies suggest that nonresorbing bone cells such as osteoblasts and osteocytes may also be targets of BPs action [4]. It has been reported that BPs can inhibit glucocorticoid-induced apoptosis in these bone cells, a process that appears to be mediated by $\mathrm{Cx} 43$ hemichannel aperture [5]. In fact, $\mathrm{Cx} 43$ is required for the antiapoptotic effect of BPs on bone cells, both in vitro and in vivo [6,7].

During aging and postmenopausal osteoporosis there is an increase in osteoclastic activity, as well as a shift in the progression of bone marrow progenitor cells (BMPC) towards an adipocytic phenotype in detriment of osteoblastogenesis [8]. This selection of adipogenesis over osteoblastogenesis in the bone marrow microenvironment has also been reported in other conditions such as type 1 Diabetes mellitus, in which there is an accumulation of bone matrix advanced glycation end products (AGEs) [9, 10]. A crucial step in the formation of mineralized tissue involves osteoblastic commitment of BMPC, followed by their mobilization to the bone surface. Maintenance of bone cell homeostatic balance depends on adequate cell-matrix and cell-cell interactions [11]. We have previously shown that high levels of AGEs accumulated on bone collagen can decrease the integrinmediated attachment of osteoblasts to the matrix [12]. We have also found that soluble and matrix-associated AGEs can impair osteoblastic growth and differentiation via activation of RAGE (receptor for AGEs), potentially contributing to diabetic osteopenia $[13,14]$. Thus, accumulation of bone AGEs has been implicated in a Diabetes-induced decrease in bone quality and/or mass, leading to an increase in fracture risk $[15,16]$.

An increase in the glycation of bone matrix proteins could be associated not only with loss of bone quality, but also with differential responses to antiosteoporotic treatments. The use of BPs for treatment of diabetic osteopathy is still an unsolved issue. Although some authors have reported a BPs-induced improvement in bone mineral density (BMD) of diabetic postmenopausal women $[17,18]$, other researchers have suggested that postmenopausal women with type 2 Diabetes could be resistant to a long-term treatment with BPs [19]. Interestingly, we have previously found that low doses $\left(10^{-8} \mathrm{M}\right)$ of alendronate can completely prevent the in vitro antiosteogenic and proapoptotic effects of AGEs on osteoblastic cells [20]. This effect of alendronate is blocked by nifedipine, an L-type calcium channel inhibitor. Although this data provides evidence suggesting that BPs could have indirect anabolic actions on osteoblasts (i.e., blocking AGEsinduced deleterious effects on this cell type), the effect of alendronate on BMPC multilineage progression in the context of an increase in bone AGEs (such as diabetic bone) remains unknown.

In the present study we have evaluated the in vitro effect of alendronate and/or AGEs on the osteoblastogenic, adipogenic, and chondrogenic potential of BMPC obtained from control rats. We have also investigated the in vivo effect of alendronate on long-bone microarchitecture in rats with insulin-deficient Diabetes, as well as the ex vivo action of this bisphosphonate on BMPC multilineage progression (i.e., the effect of orally administered alendronate on the osteogenic, adipocytic, and chondrogenic potential of BMPC obtained ex vivo from treated animals). In addition, possible mechanisms of action have been studied.

\section{Materials and Methods}

2.1. Materials. Alendronate [1-hydroxy-3-aminobutylidene1, 1-bisphosphonic acid] was kindly provided by Dr. Hector Ostrowski of Elea Laboratories (Argentina). Dulbecco's modified Eagle's medium (DMEM) was obtained from Invitrogen (Buenos Aires, Argentina), trypsin-EDTA was from Gibco and fetal bovine serum was obtained from Natocor (Córdoba, Argentina). Tissue culture disposable material was from Nunc (Tecnolab, Buenos Aires, Argentina). Centricon $10 \mathrm{kDa}$ cutoff filter cartridges were purchased from Amicon Inc. (Beverly, MA, USA). Bovine serum albumin, D-glycolaldehyde, Triton X-100 and Sirius Red dye were obtained from Sigma-Aldrich (Buenos Aires, Argentina). Dihydrorhodamine 123 (DHR) was from Molecular Probes (Buenos Aires, Argentina). All other chemicals and reagents were purchased from commercial sources and were of analytical grade.

2.2. Preparation of Advanced Glycation End Products. AGEsmodified bovine serum albumin (BSA) was prepared by incubation of $10 \mathrm{mg} / \mathrm{mL}$ BSA with $33 \mathrm{mM}$ D-glycolaldehyde in $150 \mathrm{mM}$ phosphate-buffered saline $\mathrm{pH} 7.4$ at $37^{\circ} \mathrm{C}$ for 3 days under sterile conditions, as we have previously described [20]. Control BSA was incubated in the same conditions without sugar. Unincorporated sugar was removed by centrifugation/filtration with Centricon filter cartridges. The formation of AGEs was assessed by fluorescence emission at $420 \mathrm{~nm}$ upon excitation at $340 \mathrm{~nm}$. The estimated levels of AGEsBSA obtained by this in vitro incubation were $16.9 \%$ relative fluorescence intensity/mg protein, in contrast to $3.7 \%$ for control BSA.

2.3. Animal Treatments. Three-month-old male SpragueDawley rats (190-210 g) were used. Animals were maintained in a temperature-controlled room at $23^{\circ} \mathrm{C}$, with a fixed $12 \mathrm{~h}$ light: $12 \mathrm{~h}$ darkness cycle, and fed standard rat laboratory chow and water ad libitum. All experiments on animals were done in conformity with the Guidelines on Handling and Training of Laboratory Animals published by the Universities Federation for Animals Welfare [21]. Approval for animal studies was obtained from our institutional animal welfare committee (Protocol Number 001-05-15).

For in vitro studies, control (nondiabetic untreated) animals were used as a source of BMPC. Briefly, they were sacrificed under anaesthesia by rapid cervical dislocation. Bone marrow cells were collected by flushing the femoral diaphysis medullary canal with Dulbecco's modified essential medium (DMEM) under sterile conditions and processed as described in the following sections.

To perform in vivo and ex vivo studies, 16 animals were submitted to i.p injection of nicotinamide $(50 \mathrm{mg} / \mathrm{kg}$ in physiological saline) followed by i.p. streptozotocin ( $60 \mathrm{mg} / \mathrm{kg}$ freshly dissolved in $0.05 \mathrm{M}$ citrate buffer $\mathrm{pH} 4.5$ ) in order to induce partially insulin-deficient Diabetes mellitus 
$[22,23]$, while another group of 16 rats was allowed to remain nondiabetic. A week later, nonfasting blood glucose (Wiener, Rosario, Argentina) and insulin (ALPCO, USA) were assayed according to instructions of the manufacturer, in order to verify the metabolic status of animals. Control animals showed the following results: $1.35 \pm 0.15 \mathrm{ng} / \mathrm{mL}$ for insulin and $171 \pm 8 \mathrm{mg} / \mathrm{dL}$ for glucose. Results for diabetic animals were $0.25 \pm 0.09 \mathrm{ng} / \mathrm{mL}$ for insulin and $433 \pm 22 \mathrm{mg} / \mathrm{dL}$ for glucose. Rats were then subdivided into four groups of eight animals per group: control (nontreated nondiabetic) (C) and untreated diabetic rats (D) received water ad libitum, whereas alendronate-treated nondiabetic (CA) and alendronate-treated diabetic (DA) rats received $1 \mathrm{mg} / \mathrm{kg} / \mathrm{day}$ of alendronate (Elea Lab., Buenos Aires, Argentina) in their drinking water for 2 weeks. After all treatments, nonfasting blood samples were taken and serum was stored at $-20^{\circ} \mathrm{C}$ until biochemical evaluation. Commercial kits were used to measure serum glucose, triglycerides, cholesterol, and fructosamine. All rats were sacrificed under anaesthesia by rapid cervical dislocation, after which one femur of each animal was processed for BMPC isolation and the other for histomorphometric evaluation, as described in the following sections.

2.4. Histomorphometric Examination of Long Bones. In order to evaluate the possible in vivo effect of alendronate on long bones from diabetic and nondiabetic animals, femora were processed for quantitative histomorphometric analysis. Dissected bones were fixed in $10 \%$ formalin and decalcified in $10 \%$ EDTA, embedded in paraffin, and $5 \mu \mathrm{m}$ sections were obtained with an SM 2000R Leica microtome. The sections were stained with either tartrate-resistant acid phosphatase histochemistry (TRAP) (Sigma, Buenos Aires, Argentina) to specifically identify osteoclasts or hematoxylin-eosin (H-E). Pictures were taken with a Nikon Coolpix 4500 digital camera on an Eclipse E400 Nikon microscope. Images were analyzed using the Image J program with a microscope scale plugin. In all experimental groups, microarchitecture of the proximal femoral metaphysis was evaluated by $\mathrm{H}-\mathrm{E}$ (relative trabecular volume, bone osteocytic density, and bone marrow adipocytic density) $250 \mu \mathrm{m}$ distal from the cartilage growth plate. Osteoclastic density was determined in the secondary spongiosa and calculated as positive TRAP area per square millimeter $\left(\mathrm{Oc} / \mathrm{mm}^{2}\right)$.

2.5. BMPC Isolation and Processing. Bone marrow progenitor cells were obtained as we have described previously [24]. As stated above, BMPC for in vitro experiments were derived from untreated nondiabetic animals, while those for ex vivo studies were obtained from C, D, CA, and DA groups. In each case, unfractioned bone marrow cells were flushed out of the femoral diaphysis medullary canal with Dulbecco's modified essential medium (DMEM) under sterile conditions. The resulting suspension was seeded in a $25 \mathrm{~cm}^{2}$ tissue culture flask and incubated in DMEM supplemented with penicillin $(100 \mathrm{UI} / \mathrm{mL})$, streptomycin $(100 \mathrm{mg} / \mathrm{mL})$, and $10 \%$ fetal bovine serum (FBS) (Natocor, Córdoba, Argentina) (basal medium) at $37^{\circ} \mathrm{C}$ in a humidified atmosphere with $5 \% \mathrm{CO}_{2}$ and $95 \%$ air. Nonadherent cells were removed by changing the medium after 24 hours. The culture medium was changed twice a week. When cells reached confluence (after 10 to 15 days), the cell monolayer was detached using $0.12 \%$ trypsin and $1 \mathrm{mM}$ EDTA and subcultured in tissue culture plates. Cells were then cultured as described below in different media, in order to induce their commitment and progression in a lineage-specific manner.

In the case of in vitro experiments, either control BSA or AGEs-BSA (with or without $10^{-8} \mathrm{M}$ alendronate) was added to each differentiation medium during the last 7 days of culture (for osteogenic and chondrogenic differentiation) or during the last 3 days (for adipogenic differentiation).

2.6. Osteogenic Differentiation of BMPC. BMPC were plated at a density of $5 \times 10^{4}$ cells/well in basal medium in 24-well plates and cultured until confluence. Subsequently, they were induced to differentiate to osteoblasts using an osteogenic medium (DMEM-10\% FBS containing $25 \mathrm{mg} / \mathrm{mL}$ ascorbic acid and $5 \mathrm{mM}$ sodium beta-glycerol-phosphate) [24]. Osteoblastic differentiation was evaluated after 15 days of culture by measuring specific alkaline phosphatase activity (ALP) and type 1 collagen production and after 21 days by examination of extracellular calcium deposition.

For ALP evaluation [13], cell monolayers were washed with phosphate-buffered saline (PBS) and lysed with $250 \mu \mathrm{L} \quad 0.1 \%$ Triton-X100. ALP was measured with a $100 \mu \mathrm{L}$ aliquot of the lysate, by its capacity to hydrolyze p-nitrophenylphosphate ( $\mathrm{p}$-NPP) into p-nitrophenol ( $\mathrm{p}-\mathrm{NP})$ at $37^{\circ} \mathrm{C}$ for 1 hour. The absorbance of $\mathrm{p}$-NP was recorded at $405 \mathrm{~nm}$. Aliquots of the same extract were used for protein determination by Bradford's technique [25]. Type 1 collagen production was evaluated as reported previously [24]. Briefly, cell monolayers were fixed with Bouin's solution and stained with Sirius red dye for 1 hour. The stained material was dissolved in $1 \mathrm{~mL} 0.1 \mathrm{~N}$ sodium hydroxide, and the absorbance of the solution was recorded at $550 \mathrm{~nm}$. Extracellular calcium deposits (mineralization nodules) were evaluated using Alizarin S red staining [24]. Stained calcium deposits were extracted with $1 \mathrm{~mL} 0.1 \mathrm{~N}$ sodium hydroxide, recording the optical density at $548 \mathrm{~nm}$.

2.7. Adipogenic Differentiation of BMPC. BMPC were grown to $50 \%$ confluence in 24 -well plates in basal medium. Differentiation to adipocytes was then induced as described previously [26]. Briefly, cells were incubated for 10 days with DMEM-10\% FBS supplemented with $0.5 \mathrm{mM}$ 3-isobutyl-1methylxanthine (IBMX), $1 \mathrm{mM}$ dexamethasone (Decadron, Sidus, Argentina), and $200 \mathrm{nM}$ insulin (Lilly, Buenos Aires, Argentina). At the end of this culture period the cell monolayers were lysed with $0.1 \%$ Triton-X100. Intracellular triacylglyceride levels and lipase activity were measured in the lysate, using commercial kits and according to instructions of the manufacturer (Wiener, Rosario, Argentina). Aliquots of the same extract were used for protein determination by Bradford's technique [25]. At the end of the culture period in representative experiments, differentiated 
cells were observed with a contrast-phase microscope and photographed.

2.8. Chondrogenic Differentiation of BMPC. BMPC were resuspended at $10^{7}$ cells $/ \mathrm{mL}$ in serum-free DMEM. A $10 \mu \mathrm{L}$ drop of this suspension was transferred to each well of a 24 -well plate and was incubated for 2 hours at $37^{\circ} \mathrm{C}$. After this, basal medium was added to each well and adhering cells were cultured for 24 hours. Basal medium was then discarded and chondrogenic medium was added (serum-free DMEM supplemented with $10 \mathrm{ng} / \mathrm{mL}$ TGF-b3 [Peprotech, USA], $10^{-8} \mathrm{M}$ dexamethasone, insulintransferrin-selenium (ITS) supplement [Invitrogen, USA]). Cells were cultured for an additional 21 days, changing the chondrogenic medium every three days. At the end of this culture period, chondrocytic differentiation was evaluated by determination of chondroitin-sulphate glycosaminoglycan (GAGs) accumulation, as previously described [27]. Briefly, cells were fixed, stained with $0.5 \%$ Alcian blue in $0.1 \mathrm{~N} \mathrm{HCl}$, and rinsed twice with $0.1 \mathrm{~N} \mathrm{HCl}$. Cells were finally rinsed with distilled water and Alcian blue associated with matrix GAGs was extracted with $4 \mathrm{M}$ guanidinium- $\mathrm{HCl}$, measuring absorbance at $600 \mathrm{~nm}$. In representative experiments and after staining with Alcian blue, differentiated cells were observed with a contrast-phase microscope and photographed.

\subsection{Determination of Intracellular Reactive Oxygen Species} (ROS). As a possible mechanism of action mediating the in vitro effect of alendronate and/or AGEs on BMPC obtained from untreated, nondiabetic animals, we measured the intracellular generation of ROS in BMPC by oxidation of dihydrorhodamine123 (DHR) to Rhodamine123 (Rho), as we have previously described [14]. Briefly, cells were cultured for 48 hours in DMEM under different conditions. After these incubations, culture media was replaced by phenol red-free DMEM with $10 \mu \mathrm{M}$ DHR and the cells were further incubated for 4 hours. After washing with PBS, the monolayer was lysated in $0.1 \%$ Triton X-100. The concentration of oxidized product present in the cell extract (Rho) was determined with a spectrofluorometer (excitation wavelength $495 \mathrm{~nm}$, emission wavelength $532 \mathrm{~nm}$ ).

2.10. Western Blot Analysis. BMPC from untreated, nondiabetic animals were grown to confluence in 6-well plates in DMEM-10\% FBS and then induced to differentiate to either osteoblasts or adipocytes as described above (in the presence of either BSA or AGEs-BSA, with or without $10^{-8} \mathrm{M}$ alendronate in the culture media). At the end of appropriate culture periods (15 days for osteogenic induction, 10 days for adipocytic differentiation), cells were lysed in Laemmli's buffer [28]. Lysates were heated to $100^{\circ} \mathrm{C}$ for 3 minutes, and aliquots containing $30 \mathrm{mg}$ protein were subjected to $12 \%$ SDS-PAGE. Separated proteins were transferred to PVDF membranes. After washing and blocking, the membranes were incubated overnight at $4^{\circ} \mathrm{C}$ with an antibody specific for Cbfa1/Runx2 (Santa Cruz Biotechnology, Santa Cruz, CA, USA) for evaluation of osteoblastogenesis or against PPARy (Santa Cruz Biotechnology, Santa Cruz, CA, USA) for evaluation of adipogenesis. In order to normalize results, all blots were stripped and reprobed with an anti-beta-actin antibody (Sigma, St. Louis, MO, USA). Blots were developed by an enhanced chemiluminescence method. The intensity of the specific bands was quantified by densitometry after scanning of the photographic film. Images were analyzed using the Scion beta 2 program ImageJ program (Scion Corporation, Frederick, MD, USA).

2.11. Statistical Analysis. Results are expressed as the mean \pm standard error of the mean (SEM) and were obtained from three separate experiments performed in sextuplicate. Differences between the groups were assessed by one-way analysis of variance (ANOVA) using the Tukey post hoc test. For nonnormally distributed data, the nonparametric KruskalWallis test with the Dunn post hoc test was performed using GraphPad In Stat version 3.00 (Graph Pad Software, San Diego, CA, USA). $p<0.05$ was considered significant for all statistical analyses.

\section{Results}

3.1. In Vitro Effects of AGEs and Alendronate on BMPC Multilineage Differentiation. As described above, in this first series of in vitro experiments, we evaluated the possible effects of AGEs-BSA and/or alendronate on the osteogenic, chondrogenic, and adipogenic differentiation of BMPC obtained from untreated, nondiabetic animals.

To begin with, we evaluated the effect of different concentrations of AGEs-BSA on the osteogenic potential of BMPC. Cells were cultured for one week in an osteogenic medium and then incubated for another week in the osteogenic medium plus different doses of either control BSA or AGEsBSA. At the end of this incubation period, two markers of osteoblastic differentiation (type 1 collagen production and ALP activity) were evaluated. As can be seen in Figures 1 (a) and 1 (b), $200 \mathrm{mg} / \mathrm{mL}$ of AGEs significantly decreased both parameters. Therefore, in subsequent experiments we adopted this concentration; using a similar experimental design, BMPC were submitted to a 15- or 21-day osteogenic differentiation, in which the medium was supplemented with either $200 \mathrm{mg} / \mathrm{mL}$ control BSA or AGEs-BSA in the absence or presence of alendronate $\left(10^{-9}, 10^{-8}\right.$ or $\left.10^{-7} \mathrm{M}\right)$ during the last week. 15-day cultures were used to evaluate type 1 collagen and ALP, and nodules of mineralization were analyzed in the 21-day cultures. Figures 1(c)-1(e) show that for the complete range of tested concentrations, both collagen production and ALP were stimulated by alendronate (Figures $1(\mathrm{c})$ and $1(\mathrm{~d})$ ). In addition, incubation of BMPC with AGEsBSA inhibited their ALP and collagen production at all tested alendronate concentrations. However, although AGEs reduce collagen and ALP in the presence of alendronate, their levels in these conditions are still significantly greater than those observed for control BSA without alendronate. After 21 days of osteogenic induction AGEs-BSA significantly inhibited matrix mineralization and this effect was completely prevented by $10^{-8} \mathrm{M}$ alendronate (Figure 1(e)). After 15 days of osteogenic differentiation in the conditions described 


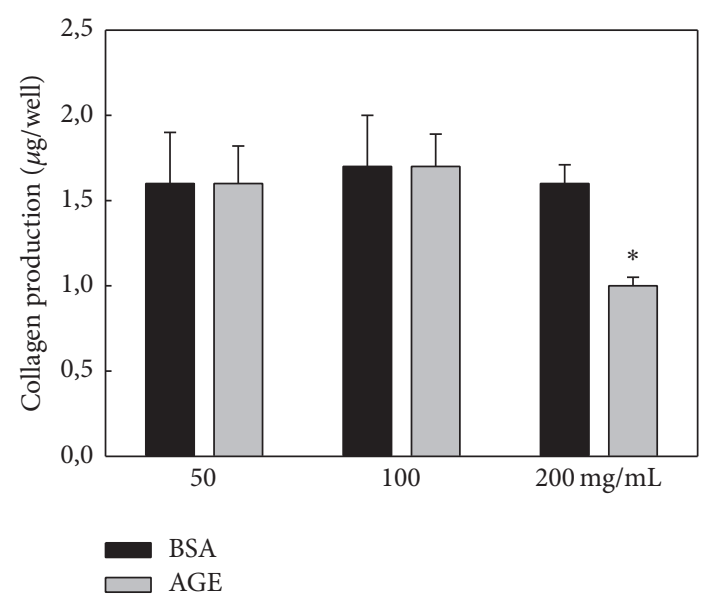

(a)

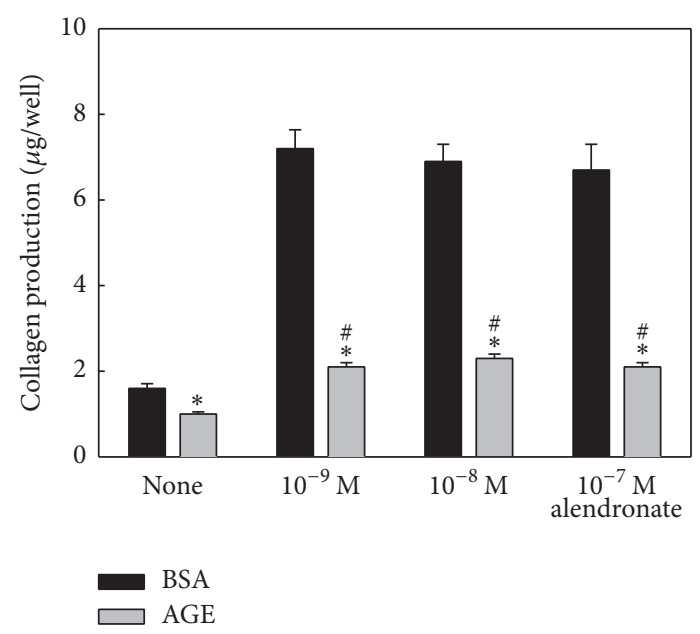

(c)

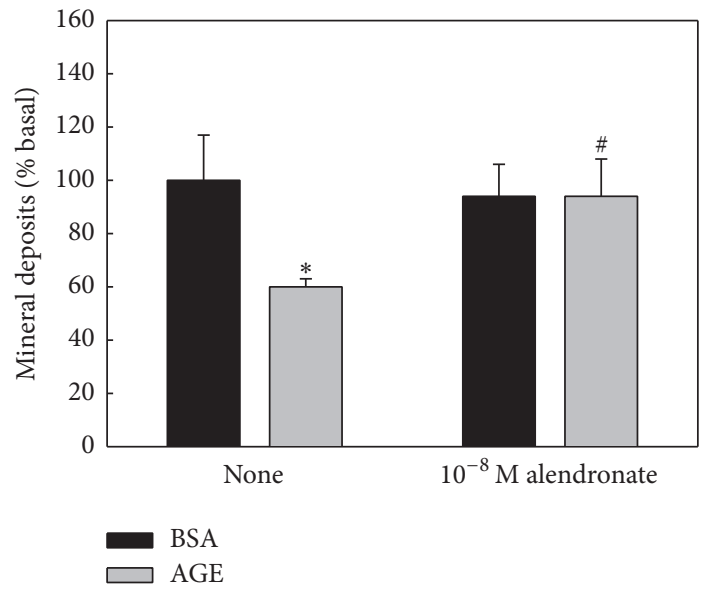

(e)

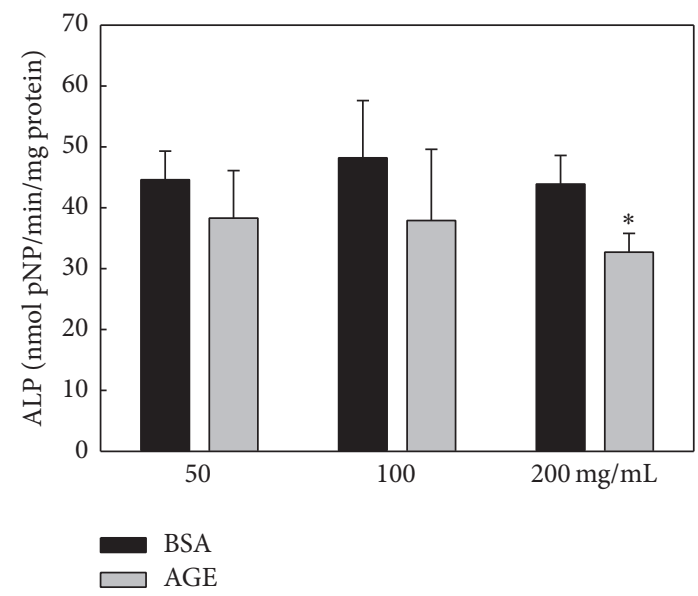

(b)

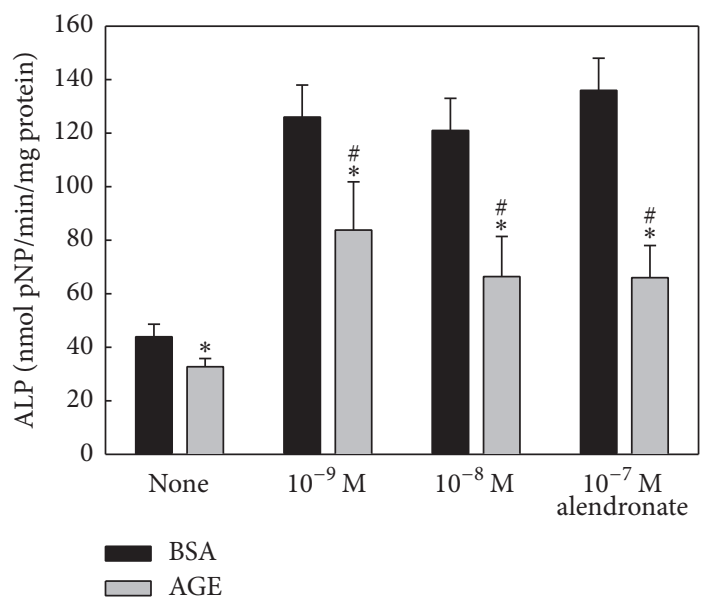

(d)

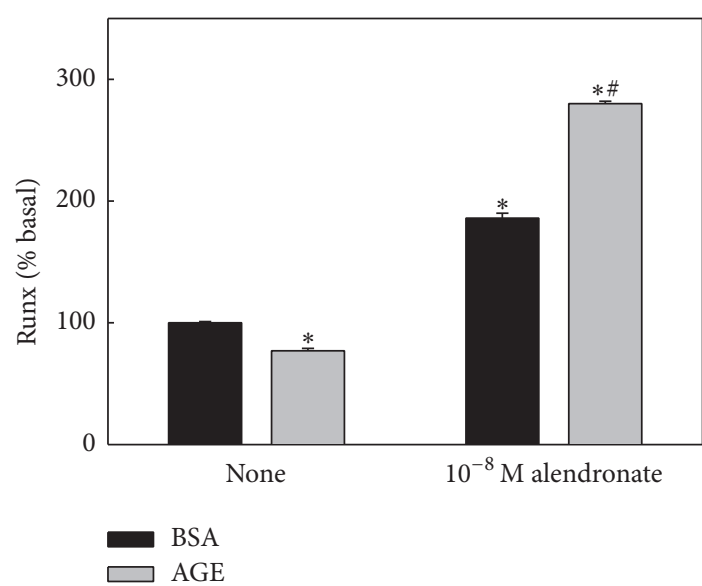

(f)

FIGURE 1: Effect of AGEs and/or alendronate on BMPC osteoblastic differentiation. BMPC were obtained from nondiabetic untreated animals and cultured in an osteogenic medium with the indicated concentrations of control BSA or AGEs-BSA (200 mg/mL if not indicated), with or without different doses of alendronate (as indicated, or $10^{-8} \mathrm{M}$ if not indicated), for either 15 days (a-d and f) or 21 days (e). Type 1 collagen production ( $\mathrm{a}$ and $\mathrm{c}$ ), alkaline phosphatase activity ( $\mathrm{b}$ and d), extracellular nodules of mineralization (e), and expression of Runx 2 by Western blot (f) were evaluated. Results are expressed as the mean \pm SEM. (a and b) ${ }^{*} p<0.05$ versus $50 \mathrm{mg} / \mathrm{mL}$ control BSA; (c-f) ${ }^{*} p<0.05$ versus control BSA, ${ }^{\#} p<0.05$ versus AGEs-BSA. 


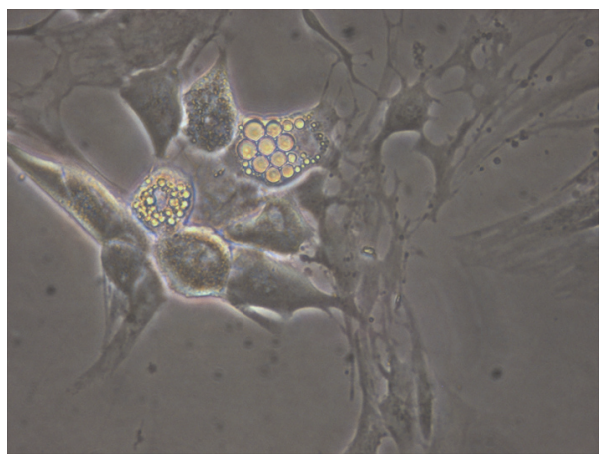

(a)

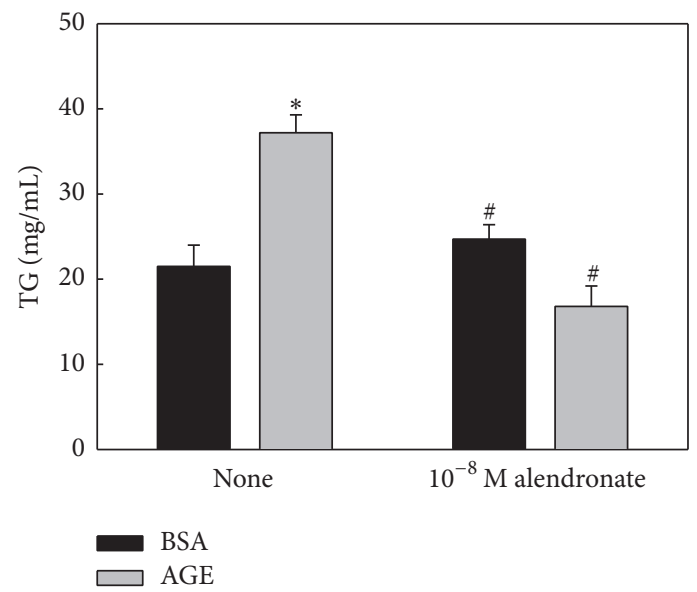

(c)

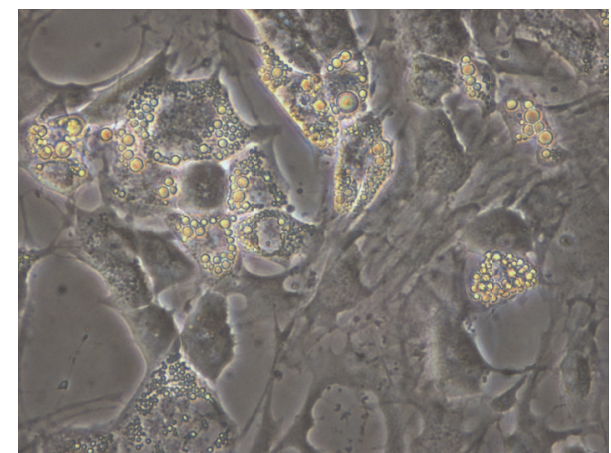

(b)

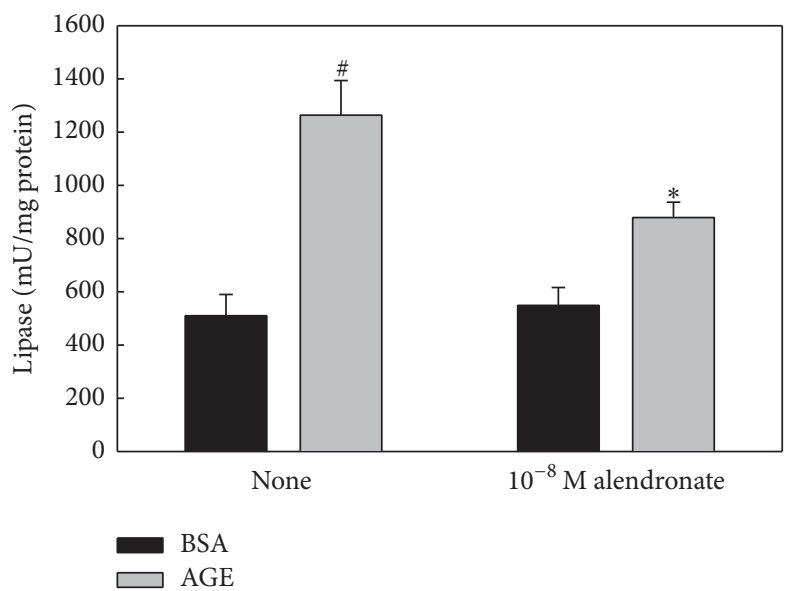

(d)

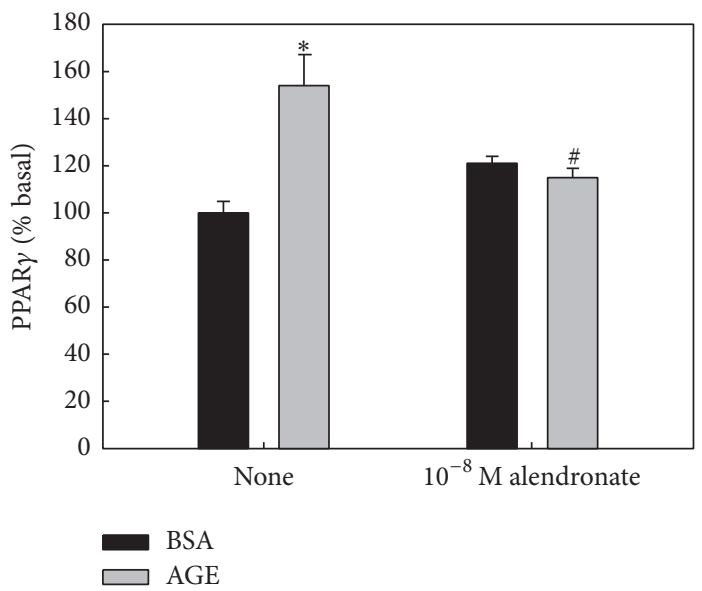

(e)

FIGURE 2: Effect of AGEs and/or alendronate on BMPC adipocytic differentiation. BMPC were obtained from nondiabetic untreated animals and cultured for 10 days in an adipogenic medium with $200 \mathrm{mg} / \mathrm{mL}$ of either control BSA or AGE-BSA, in the presence or absence of $10^{-8} \mathrm{M}$ alendronate. Contrast-phase images show intracellular lipid droplets of the monolayer exposed to control BSA (a) or AGEs-BSA (b) (Obj. 40x). Intracellular triglyceride accumulation (TG) (c), lipase enzymatic activity (d), and expression of PPAR- $\gamma$ by Western blot (e) were evaluated as markers of adipogenic differentiation. Results are expressed as the mean \pm SEM. ${ }^{*} p<0.05$ versus control BSA; ${ }^{\#} p<0.05$ versus AGEs-BSA.

above, we also evaluated the expression of the osteoblastic transcription factor Runx 2 by Western blot. As shown in Figure 1(f), AGEs-BSA on its own induced a decrease in Runx2 expression and coincubation with alendronate prevented this antiosteogenic effect. AGEs-BSA appeared to enhance the effect of alendronate on Runx2 expression.

In another set of experiments, BMPC were induced to differentiate towards an adipocytic phenotype. The adipogenic 


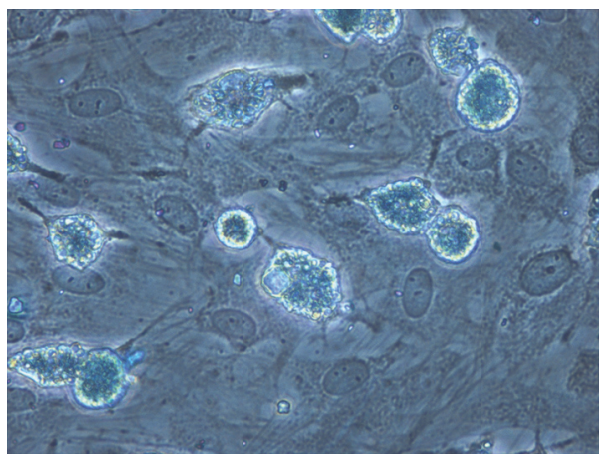

(a)

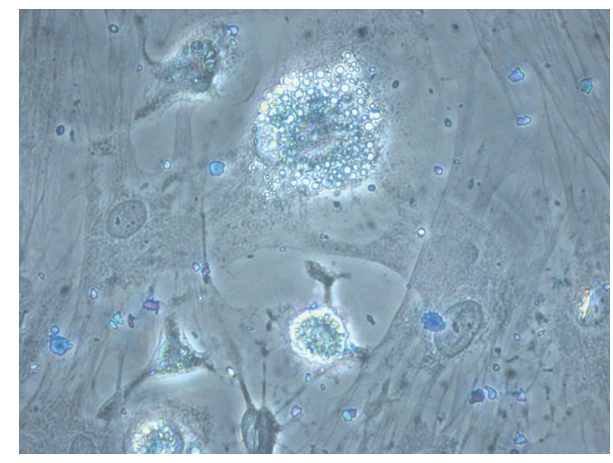

(b)

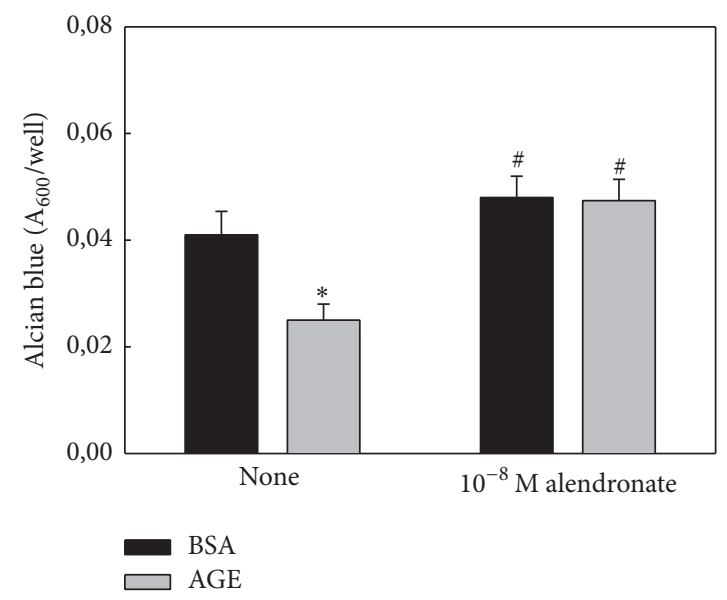

(c)

FIGURE 3: Effect of AGEs and/or alendronate on BMPC chondrocytic differentiation. BMPC were obtained from nondiabetic untreated animals and cultured for 21 days in a chondrogenic medium with $200 \mathrm{mg} / \mathrm{mL}$ of either control BSA or AGE-BSA, in the presence or absence of $10^{-8} \mathrm{M}$ alendronate. Contrast-phase images of Alcian blue staining show intracellular glycosaminoglycans (GAGs) in the monolayer exposed to control BSA (a) or AGEs-BSA (b) (Obj. 40x). GAGs-associated stain was quantitated as a marker of chondrocytic differentiation (c). Results are expressed as the mean \pm SEM. ${ }^{*} p<0.05$ versus control BSA; ${ }^{*} p<0.05$ versus AGEs-BSA.

medium was added with either control BSA or AGEs-BSA $(200 \mathrm{mg} / \mathrm{mL})$ and/or $10^{-8} \mathrm{M}$ alendronate. Contrast-phase microphotographs (Figures 2(a) and 2(b)) show that exposure of BMPC to AGEs-BSA increased their accumulation of lipid droplets. Using quantitative methods, AGEs-BSA was also found to significantly increase both intracellular triglyceride content and lipase enzymatic activity (Figures $2(\mathrm{c})$ and $2(\mathrm{~d}))$. In the same culture conditions, Western blot analysis showed that AGEs-BSA also increased the expression of the adipogenic factor PPAR- $\gamma$ (Figure 2(e)). Coincubation with alendronate prevented all of these in vitro proadipogenic effects of AGEs on BMPC (Figures 2(c)-2(e)).

In further studies, BMPC were cultured for 21 days in a chondrogenic medium with the addition of either control BSA or AGEs-BSA, in the presence or absence of alendronate. In all cases, chondrocytic differentiation was evaluated by cell accumulation of Alcian blue-stainable glycosaminoglycans (GAG). Incubation with AGEs-BSA alone significantly decreased cell-associated GAG content. This was observed in contrast-phase microphotographs (Figures 3(a) and 3(b)) and confirmed by quantitation of GAG-associated stain (Figure 3(c)). Coincubation with alendronate prevented the in vitro antichondrogenic effect of AGEs on BMPC (Figure 3(c)).

3.2. Effect of AGEs and Alendronate on Intracellular Production of Reactive Oxygen Species (ROS). Intracellular generation of ROS is known to mediate certain effects of AGEs, particularly those that depend on recognition of AGEs by RAGE, the receptor for AGEs. Thus, we evaluated whether this signal transduction pathway might be involved in the in vitro modulation of osteoblastic differentiation by AGEs-BSA and alendronate. To this end, BMPC were incubated for 48 hours with $200 \mathrm{mg} / \mathrm{mL}$ of either BSA or AGEs-BSA with or without $10^{-8} \mathrm{M}$ alendronate and in the presence or absence of $50 \mu \mathrm{M}$ vitamin $\mathrm{E}$ (as an antioxidant). After this incubation period, intracellular ROS production was determined by the dihydrorhodamine123 method. Figure 4 shows that although intracellular ROS production was significantly greater in the presence of AGEs-BSA alone, this AGEs-induced increase 


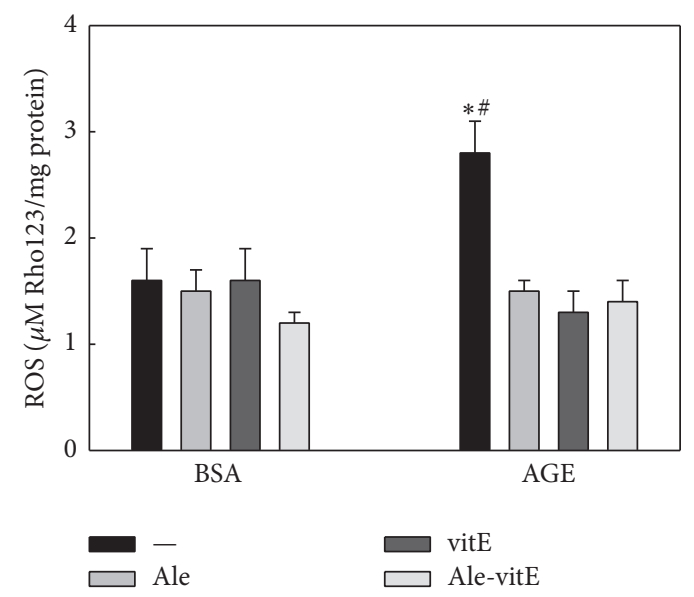

FIGURE 4: Effect of AGEs, alendronate, and vitamin $\mathrm{E}$ on the intracellular production of reactive oxygen species (ROS). BMPC were obtained from nondiabetic untreated animals and cultured for 48 hours with $200 \mathrm{mg} / \mathrm{mL}$ of either control BSA or AGEs-BSA, with or without $10^{-8} \mathrm{M}$ alendronate, in the presence or absence of $50 \mu \mathrm{M}$ vitamin E. ROS production was assessed by the Rhodamine123 method. Results are expressed as the mean \pm SEM. ${ }^{*} p<0.05$ versus control BSA; ${ }^{*} p<0.05$ versus $\mathrm{BSA} \pm$ Ale, vitE, or Ale-vitE.

was completely prevented by coincubation with vitamin E, alendronate, or both.

3.3. Diabetes-Induced Alterations in Long-Bone Microarchitecture Are Prevented by Oral Treatment with Alendronate. Insulin-deficiency was induced in rats by successive injections of streptozotocin and nicotinamide [22, 23]. Evaluation of their biochemical metabolic profile confirmed the development of Diabetes; an increase in glycaemia, fructosamine, and triglyceridemia was observed (Table 1). After induction of Diabetes the animals were treated orally with alendronate, and this did not significantly modify serum biochemical parameters (Table 1).

The effect of Diabetes and/or alendronate treatment on femoral microarchitecture was evaluated by quantitative histomorphometric analysis. Decalcified bone sections were stained with $\mathrm{H}-\mathrm{E}$ and evaluated in the secondary spongiosa to determine relative trabecular area and osteocyte density, as well as bone marrow adipocyte density. In addition, TRAP staining was performed to evaluate osteoclasts on bone surfaces of the secondary spongiosa. Compared to femora from control rats, those of diabetic rats exhibited a significant decrease in their trabecular area, osteocytic density, and TRAP activity, together with a significant increase in the density of bone marrow adipocytes (Figures 5(a)-5(d)). Alendronate treatment of diabetic animals completely prevented the Diabetes-induced decrease in trabecular bone area and osteocyte number, and it partially prevented the increase in bone marrow adiposity. As expected, alendronate treatment greatly inhibited TRAP activity (Figure 5).

3.4. Diabetes-Induced Alterations in BMPC Phenotypic Commitment Are Prevented by Oral Treatment with Alendronate. The effects of insulin-deficient Diabetes and/or oral treatment with alendronate, on the osteogenic, adipogenic, and chondrogenic commitment of BMPC were also studied. BMPC were obtained from animals of all experimental groups and incubated with lineage-specific differentiation culture media.

As can be seen in Figure 6, Diabetes induced a significant decrease in markers of BMPC osteogenic and chondrogenic potential and an increase in their adipocytic induction. Specifically, BMPC obtained from diabetic animals (versus nondiabetic untreated controls) showed (a) lower ALP activity and extracellular mineral deposits (after 15 and 21 days of osteogenic differentiation, Figures 6(a) and 6(b)), (b) a decrease in glycosaminoglycan production (after 21 days of chondrogenic differentiation, Figure 6(c)), and (c) increases in both intracellular triglyceride deposits and lipase enzymatic activity (after 10 days of adipogenic differentiation, Figures 6(d) and 6(e)). Oral treatment of animals with alendronate partially or totally prevented all of these Diabetes-induced dysregulatory effects on BMPC phenotypic commitment (Figures 6(a)-6(e)).

\section{Discussion}

Diabetes mellitus (DM) and osteoporosis constitute an important burden for health care systems worldwide. Over the past 20 years a considerable body of experimental and clinical evidence has accumulated, pointing to an association between DM and bone abnormalities that include osteopenia, osteoporosis, and/or an increased incidence of low-stress fractures [29, 30]. This Diabetes-induced bone condition, also termed diabetic osteopathy, is believed to be partly due to accumulation of AGEs in the bone matrix. AGEs form inadequate and irreversible cross-links between collagen molecules, altering the biomechanical properties of bone. AGEs also depress the functionality of osteoblasts and osteoclasts via recognition by their receptor RAGE, potentially inducing a decrease in bone turnover that can further increase AGEs accumulation [15, 16]. A deleterious effect of AGEs on bone marrow cells has also been demonstrated: an excess of these end products (or of closely related advanced oxidation protein products) has been shown to increase the apoptosis and decrease the migration, proliferation, and osteogenic differentiation of BMPC while increasing their adipogenic potential [31-34]. In addition, we and other researchers have found that BMPC isolated from diabetic rats show an increase in their adipocytic potential and a decrease in their bone-forming capacity that correlate with alterations observed in long-bone histomorphometry and pQCT analysis $[35,36]$. The in vitro results of our present study are compatible with those previous reports; we have found that AGEs (at doses similar to those found on proteins from skin and serum of diabetic patients) [37] decrease the osteogenic and chondrogenic differentiation while increasing the adipocytic commitment of BMPC obtained from control (nondiabetic untreated) rats. In addition, our present in vivo and ex vivo results confirm that the induction of insulindeficient Diabetes in rats impairs long-bone microarchitecture, while disrupting BMPC multiphenotype potential (decrease in osteogenesis and chondrogenesis and increase in adipogenesis). 


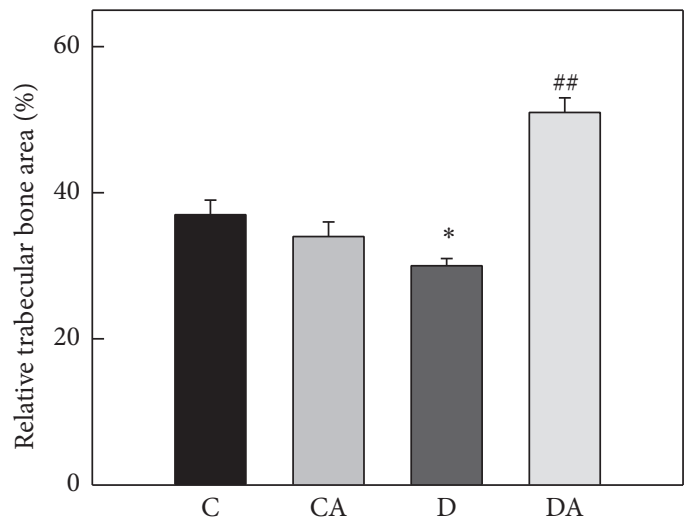

(a)

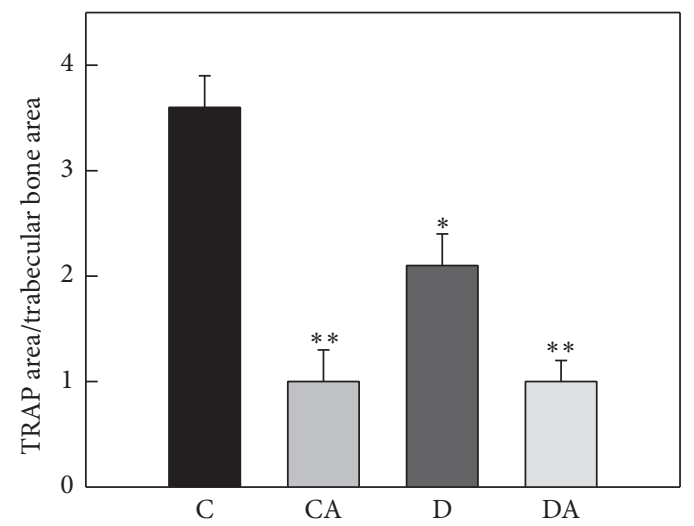

(c)

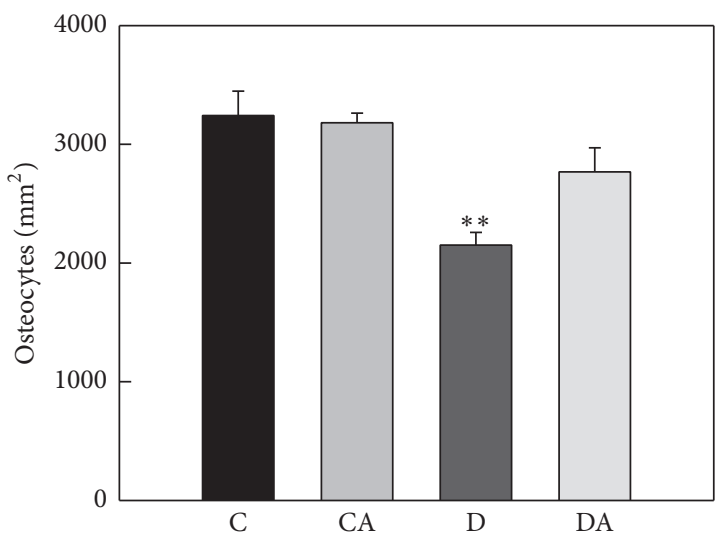

(b)

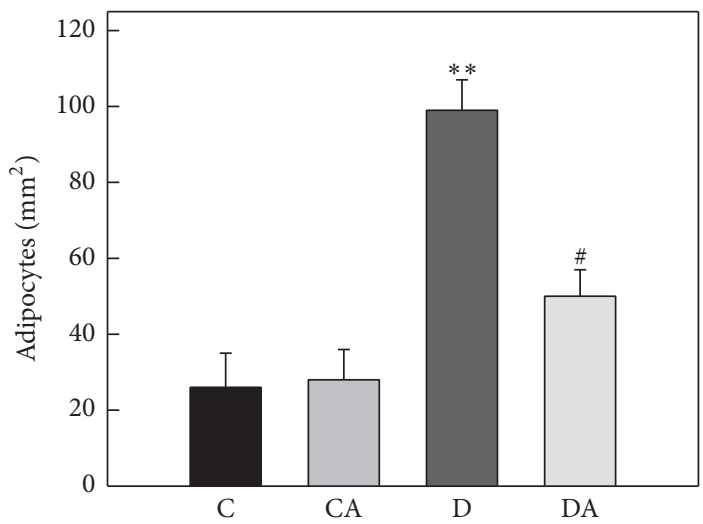

(d)

FIGURE 5: Effects of insulin-deficient Diabetes and oral treatment with alendronate on femoral microarchitecture. Diabetic and nondiabetic (control) animals were either left untreated (groups D and C) or treated for two weeks with $1 \mathrm{mg} / \mathrm{kg} /$ day of alendronate in their drinking water (groups DA and CA). Femora from animals of all groups were dissected, processed, and stained with hematoxylin-eosin or tartrateresistant acid phosphatase histochemistry (TRAP). Proximal metaphysis was analyzed by quantitative histomorphometry to determine the following: (a) trabecular bone area, (b) trabecular osteocyte density, (c) relative osteoclastic TRAP activity in the secondary spongiosa, and (d) bone marrow adipocyte density. Results are expressed as the mean \pm SEM. ${ }^{*} p<0.05$ versus $C$; ${ }^{* *} p<0.001$ versus $C$; ${ }^{*} p<0.01$ versus D; ${ }^{\# \#} p<0.001$ versus $\mathrm{D}$.

TABLE 1: Biochemical parameters in nonfasting serum obtained from animals of all experimental groups.

\begin{tabular}{lcccc}
\hline Serum parameter & Control & Control + alendronate & Diabetic & Diabetic + alendronate \\
\hline Glucose $[\mathrm{mg} / \mathrm{dL}]$ & $168 \pm 6$ & $175 \pm 9$ & $453 \pm 40^{*}$ & $380 \pm 33^{\# \&}$ \\
Triglycerides $[\mathrm{mg} / \mathrm{dL}]$ & $62 \pm 7$ & $54 \pm 4$ & $134 \pm 42^{\#}$ & $128 \pm 19^{\#}$ \\
Fructosamine $[\mu \mathrm{mol} / \mathrm{L}]$ & $120 \pm 15$ & $135 \pm 13$ & $190 \pm 12^{\%}$ & $210 \pm 16^{\%}$ \\
Cholesterol $[\mathrm{mg} / \mathrm{dL}]$ & $37 \pm 0.8$ & $37 \pm 1$ & $51 \pm 5^{\#}$ & $56 \pm 7^{\#}$ \\
\hline
\end{tabular}

Results are expressed as the mean $\pm \mathrm{SEM}, n=8$.

Differences versus C: ${ }^{\#} p<0.05 ;{ }^{\%} p<0.01$; and ${ }^{*} p<0.001$.

Differences versus D: ${ }^{\circledR} p<0.01$.

Alendronate is the most widely used agent for treatment of postmenopausal osteoporosis. This N-BP with high affinity for bone matrix shows mostly antiresorptive effects through its inhibitory action on the mevalonate pathway of osteoclasts. By this mechanism it reduces osteoclastic stress fiber and focal adhesion density, leading to a disruption of the actin cytoskeleton $[38,39]$. However, low doses of alendronate have also been shown to stimulate osteoblast function in vitro $[20,40]$ and to modulate BMPC phenotypic induction by increasing osteogenesis and decreasing adipogenesis [41-44]. Our present results are in agreement with these previous studies: in vitro exposure of BMPC to alendronate alone (at doses that have been reported in the serum and resorption lacunae of patients treated with this N-BP) [45-47] increased their osteoblastic differentiation.

It is generally accepted that treatment with alendronate increases bone mineral density (BMD) and decreases fracture incidence; however, it also reduces bone tissue remodeling 


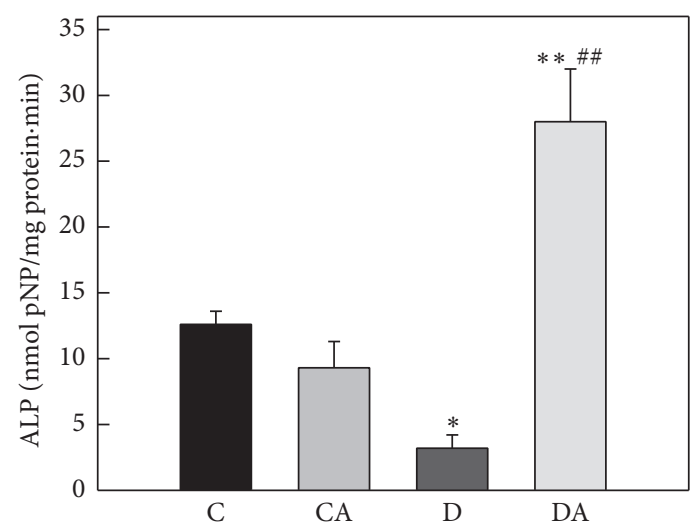

(a)

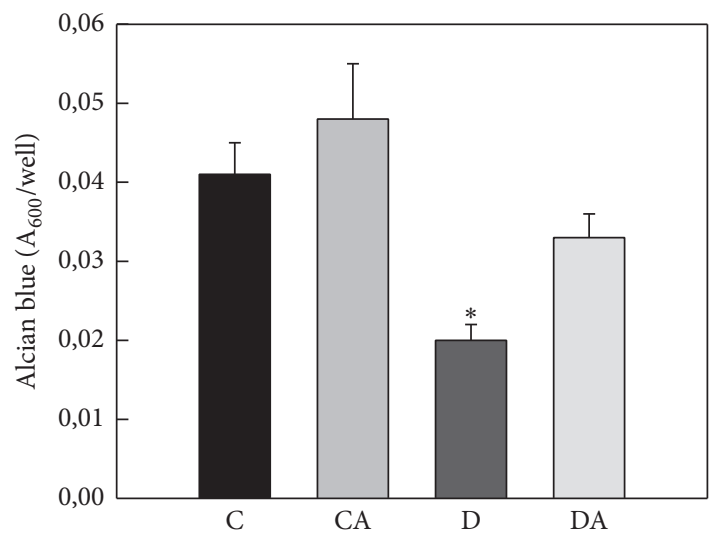

(c)

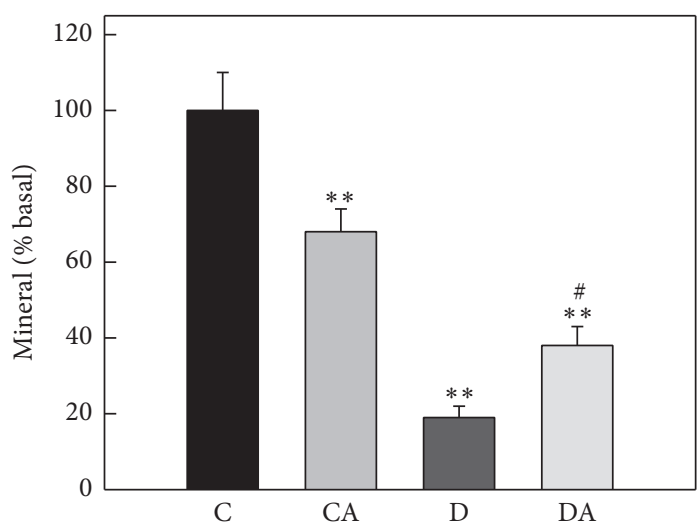

(b)

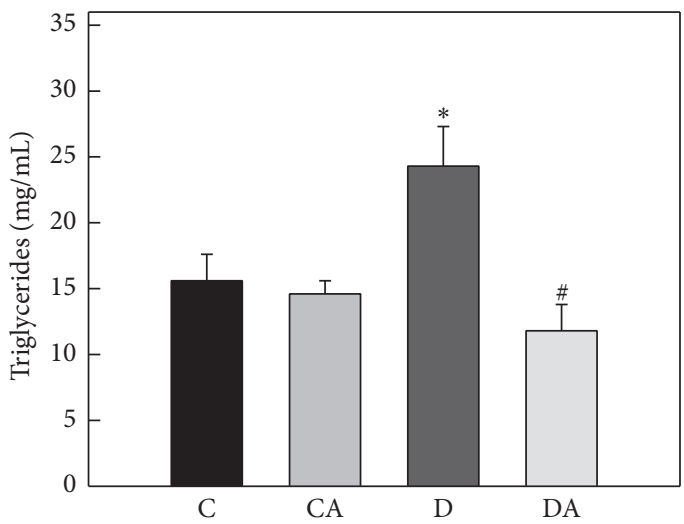

(d)

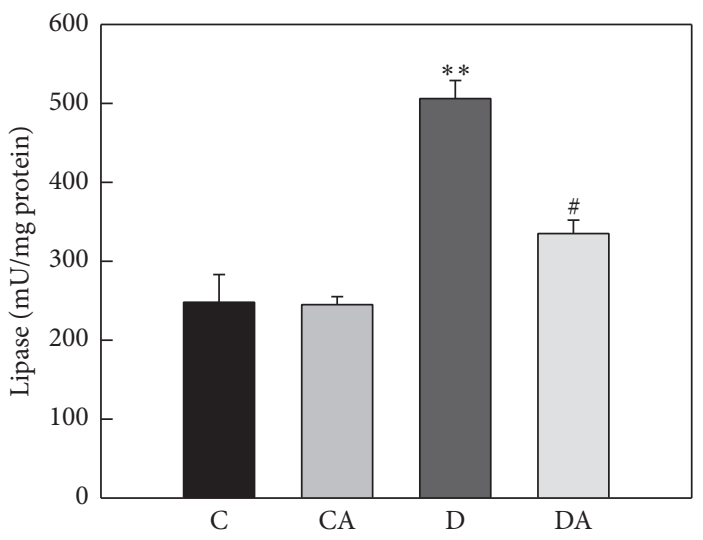

(e)

FIGURE 6: Effects of insulin-deficient Diabetes and oral treatment with alendronate on BMPC osteogenic, chondrogenic, and adipogenic potential. Diabetic and nondiabetic (control) animals were either left untreated (groups D and C) or treated for two weeks with $1 \mathrm{mg} / \mathrm{kg} / \mathrm{day}$ of alendronate in their drinking water (groups DA and CA). BMPC were obtained from animals of all experimental groups and initially cultured in a basal medium. After confluence, BMPC were replated and submitted to lineage-specific culture conditions: (a) an osteogenic medium for 15 days to evaluate alkaline phosphatase enzymatic activity; (b) an osteogenic medium for 21 days to determine extracellular mineral nodule deposits; (c) a chondrogenic medium for 21 days to quantitate GAGs-associated Alcian blue staining; and an adipogenic medium for 10 days to measure intracellular triglyceride accumulation; (d) and lipase enzymatic activity (e). Results are expressed as the mean \pm SEM. ${ }^{*} p<0.05$ versus $C ;{ }^{* *} p<0.001$ versus $C ;{ }^{\#} p<0.05$ versus $D ;{ }^{\# \#} p<0.001$ versus $D$.

that could potentially lead to an accumulation of microdamage, adversely affecting energy dissipation capabilities and overall toughness of bone [48]. This reduction in bone remodeling would also be expected to increase accumulation of AGEs, and this was investigated by other researchers in nondiabetic dogs treated for a year with different doses of alendronate. They found an increase in bone brittleness and in bone matrix accumulation of AGEs at high doses of 
alendronate, but not at doses equivalent to those used for the treatment of postmenopausal osteoporosis [48]. Since Diabetes per se may also decrease bone turnover, the treatment option of diabetic osteopathy with alendronate must be closely analyzed in order to determine its pertinence and efficacy (particularly when long-term use of this $\mathrm{N}$ BP is being considered). Through in vitro studies, we have previously demonstrated that while alendronate is able to prevent the antiosteogenic and proapoptotic effects of AGEs on osteoblasts [20], AGEs and alendronate potentiate each other to inhibit osteoclastic recruitment and activity [39]. In a clinical setting, two studies support the use of alendronate in diabetic patients: on one hand, a 3-year treatment with alendronate was associated with increased BMD in older women with type 2 Diabetes mellitus [17], whereas treatment with this N-BP for 5 years inhibited the decrease in radial BMD induced by Diabetes [18]. On the other hand, other researchers have warned that postmenopausal women with type 2 Diabetes could be resistant to long-term treatment with alendronate [19].

In view of this controversy, we have proposed the main hypothesis of the present study which is as follows: the deleterious changes in BMPC phenotypic commitment induced by AGEs accumulated in the bone matrix of an individual with Diabetes can be prevented by the presence of therapeutic doses of alendronate in the bone marrow microenvironment. In addition, these proosteogenic effects of alendronate on BMPC multilineage potential, in the context of diabetic osteopathy, will improve bone microarchitecture and functionality. Our present in vitro results support this hypothesis: we have found that exposure of control BMPC to AGEs induces a decrease in their expression of the osteoblast-specific transcription factor Runx2, type I collagen secretion, ALP activity, and extracellular mineral nodule deposition. However, when AGEs were coincubated with alendronate this antiosteogenic effect was completely abrogated. Similar results were observed when BMPC were induced to differentiate into chondrocytes: incubation with AGEs decreased their chondrocytic commitment (i.e., GAG production), whereas coincubation of AGEs with alendronate prevented this deleterious effect. On the contrary, the adipocytic differentiation of BMPC was potentiated by AGEs (increased expression of the proadipogenic regulator, PPAR- $\gamma$, of intracellular triglycerides and of lipase activity), an effect that was inhibited when AGEs were coincubated with alendronate.

This study's hypothesis is also supported by the results of our in vivo and ex vivo experiments. As stated above, development of partially insulin-deficient Diabetes in rats induced significant alterations in the microarchitecture of long bones (decreases in femoral metaphysis trabecular bone area, osteocyte density, and osteoclast TRAP activity and an increase in bone marrow adiposity). In addition, BMPC isolated from femora of diabetic rats showed a decrease in their osteogenic and chondrogenic potential and an increase in their capacity for adipogenesis. However, oral treatment of diabetic animals with alendronate prevented all of these Diabetes-induced effects on femoral microarchitecture and BMPC multilineage progression.
Intracellular ROS production is known to mediate various RAGE-dependent effects of AGEs. In an attempt to define molecular mechanisms that could be operating for both AGEs and BPs, in our present study we evaluated the intracellular production of ROS in vitro, with control $\mathrm{BMPC}$, and found that both vitamin $\mathrm{E}$ and alendronate completely curbed the AGEs-induced increase in ROS production. These results are in agreement with our previous studies with osteoblasts [20] and with reports by other authors using human umbilical vein endothelial cells $[49,50]$. RAGE-dependent intracellular ROS production is a wellknown signal transduction mechanism that leads to NF$\mathrm{KB}$ activation and expression of various subsets of genes in different cell types. However, an excessive increase in ROS production due to AGEs accumulation could induce an intracellular prooxidative state, promoting an increase in advanced oxidation protein products and ultimately cell apoptosis. This may constitute an indirect deleterious effect of AGEs on BMPC, contributing to the decrease in bone formation that has been described in experimental models of Diabetes. The in vitro results for ROS, in our present study, provide a possible mechanism of action for the in vivo preventive actions of alendronate on the antiosteogenic effects of experimental Diabetes: AGEs accumulated on bone matrix proteins exposed to hyperglycaemia can induce ROS in nearby BMPC, an effect that could be prevented by therapeutic doses of alendronate in the bone marrow microenvironment.

In conclusion, our results suggest that extracellular AGEs accumulation can alter BMPC fate by an increase in intracellular ROS production, which in turn modulates the expression of lineage-specific regulatory factors such as Runx2 and $\operatorname{PPAR} \gamma$, decreasing the osteoblastogenic and chondrogenic potential of BMPC in favour of adipogenesis. Importantly, all these AGEs-induced effects on BMPC can be prevented in vitro by cotreatment with therapeutic doses of alendronate, effects that are also operative in vivo. All in all, our present findings support the results of previous reports that show a beneficial action of alendronate in patients with Diabetes mellitus and osteoporosis.

\section{Competing Interests}

The authors declare that there is no conflict of interests regarding the publication of this paper.

\section{Acknowledgments}

This work was partially supported by grants from Facultad de Ciencias Exactas, Universidad Nacional de La Plata (UNLP), Comisión de Investigaciones Científicas de la Provincia de Buenos Aires (CICPBA) and Agencia Nacional de Promoción Científica y Técnológica (prestamo BID-1728/OC-AR, PICT 1083, and PICT-2012-0053). Sara Rocío Chuguransky is a fellow of CONICET, Ana María Cortizo is a member of the Carrera del Investigador of CICPBA, and Antonio Desmond McCarthy is a part-time Professor of Facultad de Ciencias Exactas, UNLP. 


\section{References}

[1] R. G. G. Russell, "Bisphosphonates: from bench to bedside," Annals of the New York Academy of Sciences, vol. 1068, no. 1, pp. 367-401, 2006.

[2] J. R. Green, "Skeletal complications of prostate cancer: pathophysiology and therapeutic potential of bisphosphonates," Acta Oncologica, vol. 44, no. 3, pp. 282-292, 2005.

[3] F. P. Coxon, M. H. Helfrich, R. Van't Hof et al., "Protein geranylgeranylation is required for osteoclast formation, function, and survival: inhibition by bisphosphonates and GGTI-298," Journal of Bone and Mineral Research, vol. 15, no. 8, pp. 1467-1476, 2000.

[4] L. I. Plotkin, S. C. Manolagas, and T. Bellido, "Transduction of cell survival signals by connexin- 43 hemichannels," The Journal of Biological Chemistry, vol. 277, no. 10, pp. 8648-8657, 2002.

[5] L. I. Plotkin, R. S. Weinstein, A. M. Parfitt, P. K. Roberson, S. C. Manolagas, and T. Bellido, "Prevention of osteocyte and osteoblast apoptosis by bisphosphonates and calcitonin," The Journal of Clinical Investigation, vol. 104, no. 10, pp. 1363-1374, 1999.

[6] V. Lezcano, T. Bellido, L. I. Plotkin, R. Boland, and S. Morelli, "Role of connexin 43 in the mechanism of action of alendronate: dissociation of anti-apoptotic and proliferative signaling pathways," Archives of Biochemistry and Biophysics, vol. 518, no. 2, pp. 95-102, 2012.

[7] L. I. Plotkin, V. Lezcano, J. Thostenson, R. S. Weinstein, S. C. Manolagas, and T. Bellido, "Connexin 43 is required for the anti-apoptotic effect of bisphosphonates on osteocytes and osteoblasts in vivo," Journal of Bone and Mineral Research, vol. 23, no. 11, pp. 1712-1721, 2008.

[8] J. M. Gimble, S. Zvonic, Z. E. Floyd, M. Kassem, and M. E. Nuttall, "Playing with bone and fat," Journal of Cellular Biochemistry, vol. 98, no. 2, pp. 251-266, 2006.

[9] S. Botolin, M.-C. Faugere, H. Malluche, M. Orth, R. Meyer, and L. R. McCabe, "Increased bone adiposity and peroxisomal proliferator-activated receptor- $\gamma 2$ expression in type I diabetic mice," Endocrinology, vol. 146, no. 8, pp. 3622-3631, 2005.

[10] M. E. Nuttall and J. M. Gimble, "Controlling the balance between osteoblastogenesis and adipogenesis and the consequent therapeutic implications," Current Opinion in Pharmacology, vol. 4, no. 3, pp. 290-294, 2004.

[11] M. Guan, W. Yao, R. Liu et al., "Directing mesenchymal stem cells to bone to augment bone formation and increase bone mass," Nature Medicine, vol. 18, no. 3, pp. 456-462, 2012.

[12] A. D. McCarthy, T. Uemura, S. B. Etcheverry, and A. M. Cortizo, "Advanced glycation endproducts interfere with integrinmediated osteoblastic attachment to a type-I collagen matrix," The International Journal of Biochemistry \& Cell Biology, vol. 36, no. 5, pp. 840-848, 2004.

[13] A. D. McCarthy, S. B. Etcheverry, L. Bruzzone, and A. M. Cortizo, "Effects of advanced glycation end-products on the proliferation and differentiation of osteoblast-like cells," Molecular and Cellular Biochemistry, vol. 170, no. 1-2, pp. 43-51, 1997.

[14] A. D. McCarthy, S. B. Etcheverry, L. Bruzzone, G. Lettieri, D. A. Barrio, and A. M. Cortizo, "Non-enzymatic glycosylation of a type 1 collagen matrix: effects on osteoblastic development and oxidative stress," BMC Cell Biology, vol. 2, article 16, 2001.

[15] S.-I. Yamagishi, "Role of advanced glycation end products (AGEs) in osteoporosis in diabetes," Current Drug Targets, vol. 12, no. 14, pp. 2096-2102, 2011.
[16] A. D. McCarthy, M. S. Molinuevo, and A. M. Cortizo, "AGEs and bone ageing in diabetes mellitus," Journal of Diabetes and Metabolism, vol. 4, article 276, 2013.

[17] T. H. M. Keegan, A. V. Schwartz, D. C. Bauer, D. E. Sellmeyer, and J. L. Kelsey, "Effect of alendronate on bone mineral density and biochemical markers of bone turnover in type 2 diabetic women: the fracture intervention trial," Diabetes Care, vol. 27, no. 7, pp. 1547-1553, 2004.

[18] T. Ikeda and K. Iwata, "Long-term effect of alendronate on bone mineral density in postmenopausal type 2 diabetes mellitus," Journal of Diabetes and Metabolism, vol. S1, article 002, 2011.

[19] S. Dagdelen, D. Sener, and M. Bayraktar, "Influence of type 2 diabetes mellitus on bone mineral density response to bisphosphonates in late postmenopausal osteoporosis," Advances in Therapy, vol. 24, no. 6, pp. 1314-1320, 2007.

[20] M. V. Gangoiti, A. M. Cortizo, V. Arnol, J. I. Felice, and A. D. McCarthy, "Opposing effects of bisphosphonates and advanced glycation end-products on osteoblastic cells," European Journal of Pharmacology, vol. 600, no. 1-3, pp. 140-147, 2008.

[21] "Guidelines on handling and training of laboratory animals," in The Biological Council of Animal Research, Welfare Panel. Guide for the Care and Use of Laboratory Animals: Eighth Edition, U. F. A. Purl, Ed., The National Academies Press, Washington, DC, USA, 2011.

[22] A. Tahara, A. Matsuyama-Yokono, R. Nakano, Y. Someya, and M. Shibasaki, "Hypoglycaemic effects of antidiabetic drugs in streptozotocin-nicotinamide-induced mildly diabetic and streptozotocin-induced severely diabetic rats," Basic and Clinical Pharmacology and Toxicology, vol. 103, no. 6, pp. 560-568, 2008.

[23] T. Szkudelski, "Streptozotocin-nicotinamide-induced diabetes in the rat. Characteristics of the experimental model," Experimental Biology and Medicine, vol. 237, no. 5, pp. 481-490, 2012.

[24] M. S. Molinuevo, L. Schurman, A. D. McCarthy et al., "Effect of metformin on bone marrow progenitor cell differentiation: in vivo and in vitro studies," Journal of Bone and Mineral Research, vol. 25, no. 2, pp. 211-221, 2010.

[25] M. M. Bradford, "A rapid and sensitive method for the quantitation of microgram quantities of protein utilizing the principle of protein-dye binding," Analytical Biochemistry, vol. 72, no. 1-2, pp. 248-254, 1976.

[26] J. I. Felice, M. V. Gangoiti, M. S. Molinuevo, A. D. McCarthy, and A. M. Cortizo, "Effects of a metabolic syndrome induced by a fructose-rich diet on bone metabolism in rats," Metabolism: Clinical and Experimental, vol. 63, no. 2, pp. 296-305, 2014.

[27] S. Bahmanpour and D. F. Paulsen, "Inhibition of chondrogenic differentiation in chick limb-bud mesenchyme microcultures treated with cyclosporine," Indian Journal of Pharmacology, vol. 38, no. 1, pp. 43-48, 2006.

[28] U. K. Laemmli, "Cleavage of structural proteins during the assembly of the head of bacteriophage T4," Nature, vol. 227, no. 5259, pp. 680-685, 1970.

[29] K. Wongdee and N. Charoenphandhu, "Osteoporosis in diabetes mellitus: possible cellular and molecular mechanisms," World Journal of Diabetes, vol. 2, no. 3, pp. 41-48, 2011.

[30] J. N. Farr and S. Khosla, "Determinants of bone strength and quality in diabetes mellitus in humans," Bone, vol. 82, pp. 2834, 2016.

[31] S. Kume, S. Kato, S.-I. Yamagishi et al., "Advanced glycation endproducts attenuate human mesenchymal stem cells and prevent cognate differentiation into adipose tissue, cartilage, and bone," 
Journal of Bone and Mineral Research, vol. 20, no. 9, pp. 16471658, 2005.

[32] K. Yang, X. Q. Wang, Y. S. He et al., "Advanced glycation end products induce chemokine/cytokine production via activation of $\mathrm{p} 38$ pathway and inhibit proliferation and migration of bone marrow mesenchymal stem cells," Cardiovascular Diabetology, vol. 9, article 66, 2010.

[33] N. Sun, L. Yang, Y. Li et al., "Effect of advanced oxidation protein products on the proliferation and osteogenic differentiation of rat mesenchymal stem cells," International Journal of Molecular Medicine, vol. 32, no. 2, pp. 485-491, 2013.

[34] E. Weinberg, T. Maymon, and M. Weinreb, "AGEs induce caspase-mediated apoptosis of rat BMSCs via TNF $\alpha$ production and oxidative stress," Journal of Molecular Endocrinology, vol. 52, no. 1, pp. 67-76, 2014.

[35] A. Stolzing, D. Sellers, O. Llewelyn, and A. Scutt, "Diabetes induced changes in rat mesenchymal stem cells," Cells Tissues Organs, vol. 191, no. 6, pp. 453-465, 2010.

[36] M. J. Tolosa, S. R. Chuguransky, C. Sedlinsky et al., "Insulindeficient diabetes-induced bone microarchitecture alterations are associated with a decrease in the osteogenic potential of bone marrow progenitor cells: preventive effects of metformin," Diabetes Research and Clinical Practice, vol. 101, no. 2, pp. 177186, 2013.

[37] S. Genuth, W. Sun, P. Cleary et al., "Skin advanced glycation end products glucosepane and methylglyoxal hydroimidazolone are independently associated with long-Term microvascular complication progression of type 1 diabetes," Diabetes, vol. 64, no. 1, pp. 266-278, 2015.

[38] N. H. Kazmers, S. A. Ma, T. Yoshida, and P. H. Stern, "Rho GTPase signaling and PTH 3-34, but not PTH 1-34, maintain the actin cytoskeleton and antagonize bisphosphonate effects in mouse osteoblastic MC3T3-E1 cells," Bone, vol. 45, no. 1, pp. 5260, 2009.

[39] M. V. Gangoiti, P. S. Anbinder, A. M. Cortizo, and A. D. McCarthy, "Morphological changes induced by advanced glycation endproducts in osteoblastic cells: effects of co-incubation with alendronate," Acta Histochemica, vol. 115, no. 7, pp. 649657, 2013.

[40] M. Tsuchimoto, Y. Azuma, O. Higuchi et al., "Alendronate modulates osteogenesis of human osteoblastic cells in vitro," Japanese Journal of Pharmacology, vol. 66, no. 1, pp. 25-33, 1994.

[41] G. Duque and D. Rivas, "Alendronate has an anabolic effect on bone through the differentiation of mesenchymal stem cells," Journal of Bone and Mineral Research, vol. 22, no. 10, pp. 16031611, 2007.

[42] H. K. Kim, J. H. Kim, A. A. Abbas, and T. R. Yoon, "Alendronate enhances osteogenic differentiation of bone marrow stromal cells: a preliminary study," Clinical Orthopaedics and Related Research, vol. 467, no. 12, pp. 3121-3128, 2009.

[43] V. Ribeiro, M. Garcia, R. Oliveira, P. S. Gomes, B. Colaço, and M. H. Fernandes, "Bisphosphonates induce the osteogenic gene expression in co-cultured human endothelial and mesenchymal stem cells," Journal of Cellular and Molecular Medicine, vol. 18, no. 1, pp. 27-37, 2014.

[44] C.-H. Chang, C.-Z. Wang, J.-K. Chang, C.-Y. Hsu, and M.-L. Ho, "The susceptive alendronate-treatment timing and dosage for osteogenesis enhancement in human bone marrow-derived stem cells," PLoS ONE, vol. 9, no. 8, article e105705, 2014.

[45] N. Giuliani, M. Pedrazzoni, G. Negri, G. Passeri, M. Impicciatore, and G. Girasole, "Bisphosphonates stimulate formation of osteoblast precursors and mineralized nodules in murine and human bone marrow cultures in vitro and promote early osteoblastogenesis in young and aged mice in vivo," Bone, vol. 22, no. 5, pp. 455-461, 1998.

[46] M. J. Rogers, S. Gordon, H. L. Benford et al., "Cellular and molecular mechanisms of action of bisphosphonates," Cancer, vol. 88, supplement 12, pp. 2961-2978, 2000.

[47] G.-I. Im, S. A. Qureshi, J. Kenney, H. E. Rubash, and A. S. Shanbhag, "Osteoblast proliferation and maturation by bisphosphonates," Biomaterials, vol. 25, no. 18, pp. 4105-4115, 2004.

[48] S. Y. Tang, M. R. Allen, R. Phipps, D. B. Burr, and D. Vashishth, "Changes in non-enzymatic glycation and its association with altered mechanical properties following 1-year treatment with risedronate or alendronate," Osteoporosis International, vol. 20, no. 6, pp. 887-894, 2009.

[49] T. Okamoto, S.-I. Yamagishi, Y. Inagaki et al., "Incadronate disodium inhibits advanced glycation end products-induced angiogenesis in vitro," Biochemical and Biophysical Research Communications, vol. 297, no. 2, pp. 419-424, 2002.

[50] S. Yamagishi, T. Matsui, K. Nakamura, and M. Takeuchi, "Minodronate, a nitrogen-containing bisphosphonate, inhibits advanced glycation end product-induced vascular cell adhesion molecule-1 expression in endothelial cells by suppressing reactive oxygen species generation," International Journal of Tissue Reactions, vol. 27, no. 4, pp. 189-195, 2005. 

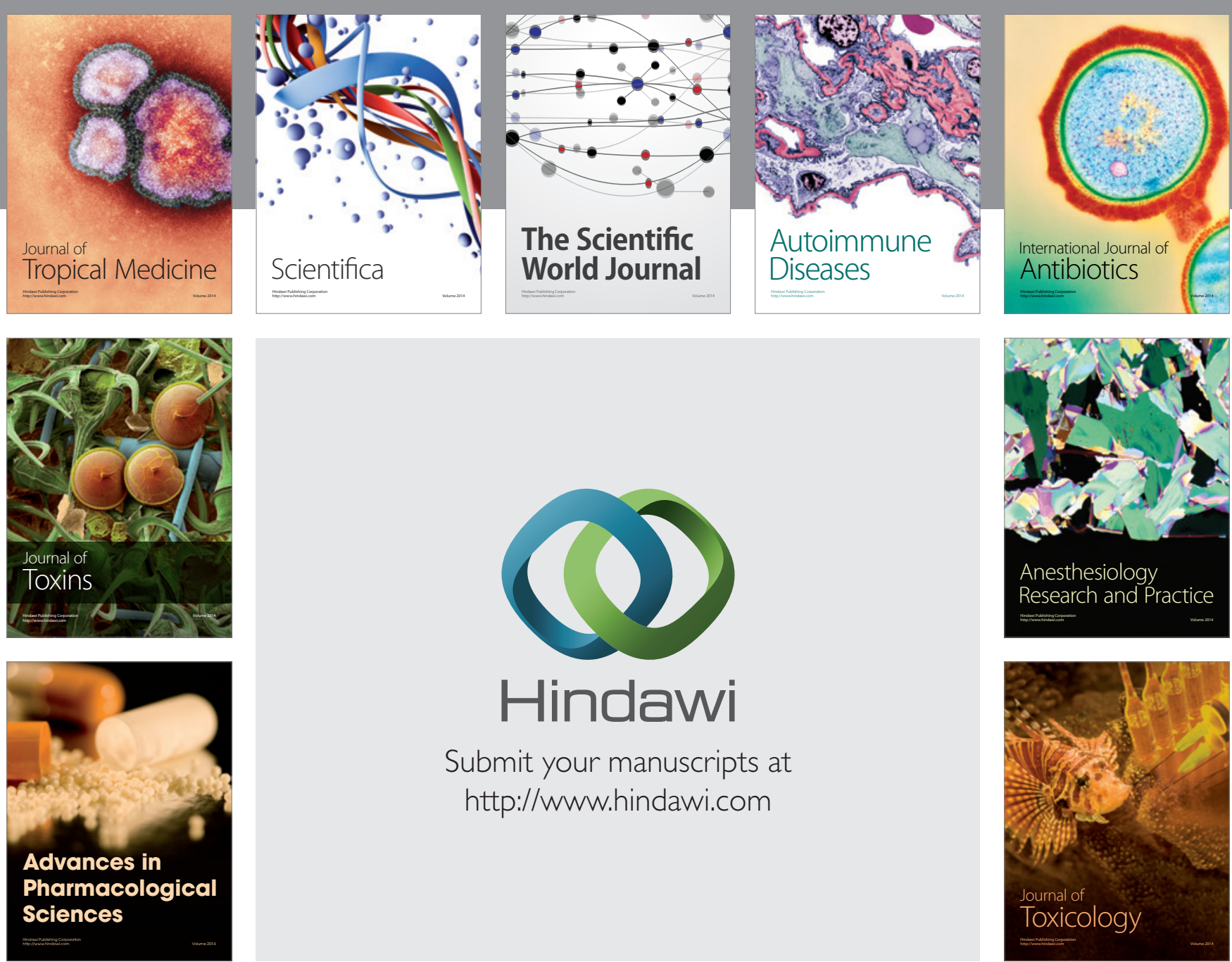

\section{Hindawi}

Submit your manuscripts at

http://www.hindawi.com
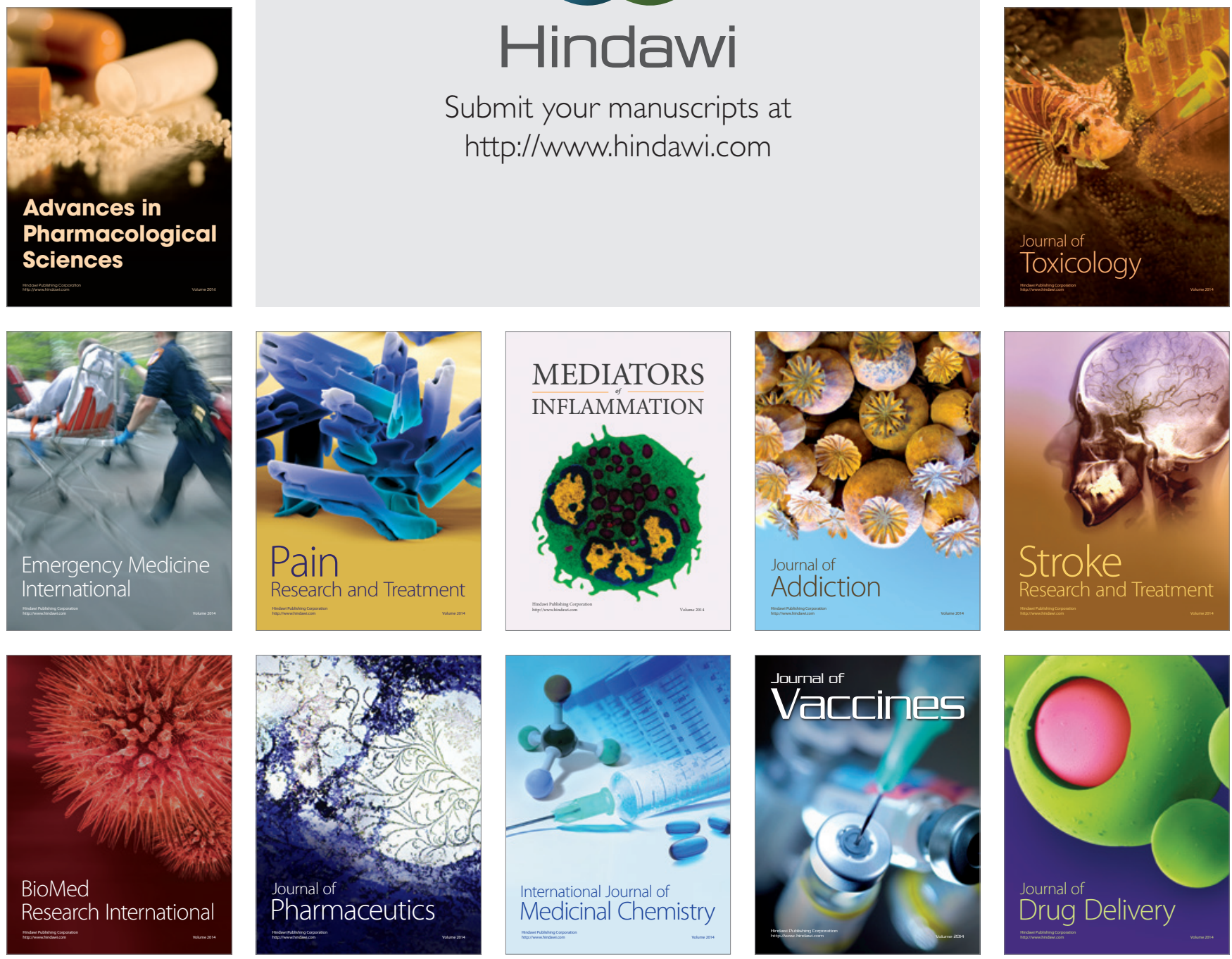